\title{
To Improve Translational Research in Subarachnoid Hemorrhage
}

\author{
Hidenori Suzuki $^{1}$ (D) $\cdot$ Fumi Nakano ${ }^{1}$
}

Received: 6 June 2017 / Accepted: 8 June 2017 / Published online: 16 June 2017

(C) Springer Science+Business Media, LLC 2017

The advantage of animal studies is to study a relatively homogeneous group of animals instead of a heterogeneous group of patients in clinical studies. Animal studies also offer a wider range of possibilities for example such as examining toxicity of a specific treatment or studying the underlying mechanisms of diseases. However, because most of new therapies shown to be effective in animal studies have been ineffective in clinical trials, some guidelines including the Stroke Therapy Academic Industry Roundtable (STAIR) were proposed to improve the quality and reproducibility of individual animal studies evaluating neuroprotective drugs in ischemic stroke [1-3]. Recent special issues "Challenges and Controversies in Translational Stroke Research, Part 1 and 2" in this journal provide an excellent overview as to the innate biological variability and the methodological challenges that are needed to address bias in preclinical research for the successful translation of experimental therapies to clinical stroke treatments. The proposed standards include (1) clinical relevance of animal models (detailed information on animals used (species, strain, age, weight, gender, etc.), selection of anesthetics, inclusion and exclusion criteria), (2) sample size calculation and accurate statistical analysis, (3) treatment (randomization, allocation concealment, dose-response determinations, therapeutic time window, blood-brain barrier permeability and tissue drug levels, physiological monitoring), (4) outcome (blinded assessment, at least two outcome measures (morphology and function), covering both acute (1-3 days) and longterm (7-30 days) endpoints), and (5) reporting of animals

Hidenori Suzuki

mie1192suzuki@gmail.com

1 Department of Neurosurgery, Mie University Graduate School of Medicine, 2-174 Edobashi, Tsu, Mie 514-8507, Japan excluded from analysis, potential conflicts of interest, and study funding [1-7]. In addition, several challenges exist to successfully translate the outcomes from animal research to humans in a clinical setting. First, age and sex are two important non-modifiable risk factors for stroke [5, 8]. With aging, there is a shift toward a proinflammatory phenotype in the brain as well as the periphery, and blood-brain barrier disruption [8]. Women are protected from stroke before menopause, but have increased stroke rates and worse outcomes at older ages [5]. On the other hand, the use of adult reproductive female animals in stroke research is complicated by the sex hormone cycle. However, the male-biased use of research animals is distinguished from the clinical situation where there is a disproportionate and growing female stroke population, making it important to include both sexes with diverse ages in preclinical as well as clinical studies that evaluate potential stroke therapies. Second, cerebrovascular anatomy and collaterals, as well as biological and secondary neuroinflammatory responses to insults, are different between species or strains, causing flawed design, unreliable outcomes, unnecessarily more costs, and experimental animals [7, 9-11]. Genetic differences between animals and humans, and even within animal species, strains, and cell lines, may affect the immune responses and outcomes $[10,12,13]$. Third, stroke patients have many comorbidities or vascular risk factors including hypertension, diabetes mellitus, dyslipidemia, cardiac diseases, current smoking, obesity, poor diet, inactivity, high alcohol intake, psychosocial stress and depression, social factors such as marital and residence status (i.e., living alone), and prestroke dysfunction, causing stroke severity [5, 14]. Animal models having these factors are also subjected to different stroke injuries or changes in the structure of the neurovascular unit [14]. Thus, the use of young healthy animals causes a barrier for translation of findings to patients, while, in preclinical stroke studies with comorbidities, comorbidity duration 
and severity as well as housing conditions of the animals used, which potentially influence outcomes, should be reported in details.

Human stroke is very complex, and no single preclinical model can comprise all the variables contributing to stroke injury and recovery. It is inappropriate to assume that targeting only one aspect of the cascade is sufficient to produce clinical benefit, and to evaluate purported neuroprotective drugs in a limited number of models in the relatively small number of laboratories. Challenging paradigms include simulation of polypharmacy and specialized large animal models [15] in addition to factors described above. Furthermore, these options should be combined, and therefore, the resulting setup is too complex to be covered by a single laboratory. To overcome the issues, a consortium of multiple laboratories is suggested to be organized for large-scale testing of the same neuroprotective drug in multiple animal stroke models in multiple species across multiple sites with rigorous performance of the necessary experiments extending the methods to include a consideration of the innate biological and methodological variability that the agent will ultimately encounter when translated to humans [16]. Multicenter phase III randomized preclinical trials aim to apply quality standards of clinical investigations and are expected to conduct confirmative research in a highly complex, but realistic environment, leading to translational research strategies $[17,18]$. Pre-clinically, multimodal imaging can noninvasively generate data on a range of parameters, allowing the animal to be recovered for subsequent behavioral testing and/or the brain taken for further molecular or histological analysis, and resulting in saving animals and costs [19]. In addition, academic-industry collaborations are needed to overcome the translational stroke research roadblock, such as the advent of the multicenter preclinical trial concept [20]. Although the guidelines stem from the need to assess neuroprotection for acute ischemic stroke, they have been applied to the evaluation of other treatments, interventions, and fields of neuroscience with or without a modification [21-26].

Aneurysmal subarachnoid hemorrhage (SAH) is a wellknown devastating cerebrovascular disease that relatively young people suffer compared with other types of stroke [27, 28]. SAH-induced brain injury is also very complex [27, 28], and more than 72 kinds of animal models have been reported [29]. The endovascular perforation animal model shows acute metabolic changes similar to clinical findings and becomes the most popular one for studies of early brain injury (EBI), a concept to explain acute pathophysiological events that occur in the brain before onset of cerebral vasospasm within the first $72 \mathrm{~h}$ of aneurysmal SAH [28]. However, both EBI and cerebral vasospasm occur in the time frame of $72 \mathrm{~h}$ in the mouse or rat model, precluding the dissociation between EBI and cerebral vasospasm-induced or other delayed brain injuries. Therefore, SAH researchers should understand the advantages and disadvantages of each animal model and choose a suitable model dependent on the research question. In addition, systematic reviews expose biases and inadequacies in the methodology of individual studies, contributing to improving translation of animal research to humans [12]. To make systemic reviews more useful, publication bias or an overestimation of the effect of a treatment has to be avoided by reporting negative and neutral results and promoting data sharing [1], and making systematic reviews of animal studies a routine is our scientific and societal responsibility, just as with clinical studies in humans not simply for transparency but also to avoid waste of financial resources and unnecessary duplication of animal studies [12]. In a SAH research, a systematic review of animal studies of endothelin receptor antagonists was reported after the clinical trials were failed [30]. The review revealed that various animal models including both sexes and primate models were used, but randomization of treatment groups and blinded assessment of outcome were performed only in the half of experiments. Furthermore, neurobehavioral scores were reported in none of the experiments, and case fatality only in $15 \%$ of experiments. The inclusion of death as an outcome measure in experimental SAH studies may be critical in determining the true efficacy of a potential therapeutic agent, because SAH is a disease with a high mortality, and therefore, excluding animals that have died from the study may skew the results so that findings are biased toward surviving animals. The authors thus concluded that there was no evidence from animal studies that treatment with an endothelin receptor antagonist improved clinically relevant outcomes after SAH, while the reduction in cerebral vasospasm observed in animal studies was consistent with that observed in clinical trials, an effect that was not associated with better functional outcome in patients [30]. The different outcomes between experimental and clinical studies may also occur due to misinterpretation of data and confused terminology and definitions [31]. Anyway, we can say that the failed clinical trials could have been predicted and avoided if the review and the meta-analysis had been performed before planning the clinical trials. The step, along with the current recommendations including STAIRs, may improve the successful translation of experimental therapies to clinical stroke treatments. Although it remains to be determined whether these approaches enhance the quality of evaluation in the preclinical settings and increase the likelihood of successful clinical trials, systematic reviews of all relevant animal experiments would be a prerequisite for the design of new clinical trials.

\section{Compliance with Ethical Standards}

Funding This study was funded by a grant-in-aid for Scientific Research from Japan Society for the Promotion of Science (grant number, 17K10825), and Mie Medical Research Foundation (grant number, N/A) to Dr. Suzuki.

Conflict of Interest The authors report no conflicts of interest. 
Ethical Approval This article does not contain any studies with human participants or animals performed by any of the authors.

\section{References}

1. Lapchak PA, Zhang JH, Noble-Haeusslein LJ. RIGOR guidelines: escalating STAIR and STEPS for effective translational research. Transl Stroke Res. 2013;4:279-85.

2. Lapchak PA, Zhang JH. Translational stroke research guideline projections: the 20/20 standards. Transl Stroke Res. 2017; doi:10. 1007/s12975-017-0534-6.

3. Marbacher S. Can quality improvement tools overcome the translational roadblock - the vital influence of the researcher. Transl Stroke Res. 2017;8:203-5.

4. Fisher M, Feuerstein G, Howells DW, Hurn PD, Kent TA, Savitz SI, et al., STAIR Group. Update of the stroke therapy academic industry roundtable preclinical recommendations. Stroke. 2009:40:2244-50

5. Ahnstedt H, McCullough LD, Cipolla MJ. The importance of considering sex differences in translational stroke research. Transl Stroke Res. 2016;7:261-73.

6. Henninger N, Fisher M. Extending the time window for endovascular and pharmacological reperfusion. Transl Stroke Res. 2016;7:284-93.

7. Hoffmann U, Sheng H, Ayata C, Warner DS. Anesthesia in experimental stroke research. Transl Stroke Res. 2016;7:358-67.

8. Lucke-Wold BP, Turner RC, Lucke-Wold AN, Rosen CL, Huber JD. Age and the metabolic syndrome as risk factors for ischemic stroke: improving preclinical models of ischemic stroke. Yale J Biol Med. 2012;85:523-39.

9. Howells DW, Porritt MJ, Rewell SS, O'Collins V, Sena ES, van der Worp HB, et al. Different strokes for different folks: the rich diversity of animal models of focal cerebral ischemia. J Cereb Blood Flow Metab. 2010;30:1412-31.

10. Becker KJ. Strain-related differences in the immune response: relevance to human stroke. Transl Stroke Res. 2016;7:303-12.

11. Liesz A, Kleinschnitz C. Regulatory T cells in post-stroke immune homeostasis. Transl Stroke Res. 2016;7:313-21.

12. Hooijmans CR, Ritskes-Hoitinga M. Progress in using systematic reviews of animal studies to improve translational research. PLoS Med. 2013;10:e1001482. doi:10.1371/journal.pmed.1001482.

13. D'Abbondanza JA, Ai J, Lass E, Wan H, Brathwaite S, Tso MK, et al. Robust effects of genetic background on responses to subarachnoid hemorrhage in mice. J Cereb Blood Flow Metab. 2016;36:1942-54.

14. Ergul A, Hafez S, Fouda A, Fagan SC. Impact of comorbidities on acute injury and recovery in preclinical stroke research: focus on hypertension and diabetes. Transl Stroke Res. 2016;7:248-60.

15. Cai B, Wang N. Large animal stroke models vs. rodent stroke models, pros and cons, and combination? Acta Neurochir Suppl. 2016;121:77-81.
16. Kent TA, Mandava P. Embracing biological and methodological variance in a new approach to pre-clinical stroke testing. Transl Stroke Res. 2016;7:274-83.

17. Dirnag1 U, Fisher M. International, multicenter randomized preclinical trials in translational stroke research: it's time to act. J Cereb Blood Flow Metab. 2012;32:933-5.

18. Boltze J, Wagner D-C, Henninger N, Plesnila N, Ayata C. Phase III preclinical trials in translational stroke research: community response on framework and guidelines. Transl Stroke Res. 2016;7: 241-7.

19. Muir KW, Macrae IM. Neuroimaging as a selection tool and endpoint in clinical and pre-clinical trials. Transl Stroke Res. 2016;7: 368-77.

20. Boltze J, Wagner D-C, Barthel H, Gounis MJ. Academic-industry collaborations in translational stroke research. Transl Stroke Res. 2016; 7:343-53.

21. Linfante I, Cipolla MJ. Improving reperfusion therapies in the era of mechanical thrombectomy. Transl Stroke Res. 2016;7:294-302.

22. Yang B, Parsha K, Schaar K, Xi XP, Aronowski J, Savitz SI. Various cell populations within the mononuclear fraction of bone marrow contribute to the beneficial effects of autologous bone marrow cell therapy in a rodent stroke model. Transl Stroke Res. 2016;7:322-30.

23. Jolkkonen J, Kwakkel G. Translational hurdles in stroke recovery studies. Transl Stroke Res. 2016;7:331-42.

24. Rodríguez-Frutos B, Otero-Ortega L, Gutiérrez-Fernández M, Fuentes B, Ramos-Cejudo J, Díez-Tejedor E. Stem cell therapy and administration routes after stroke. Transl Stroke Res. 2016;7: 378-87.

25. Hommel M, Detante O, Favre I, Touzé E, Jaillard A. How to measure recovery? Revisiting concepts and methods for stroke studies. Transl Stroke Res. 2016;7:388-94.

26. Yang Y, Kimura-Ohba S, Thompson J, Rosenberg GA. Rodent models of vascular cognitive impairment. Transl Stroke Res. 2016;7:407-14

27. Suzuki H, Shiba M, Nakatsuka Y, Nakano F, Nishikawa H. Higher cerebrospinal fluid $\mathrm{pH}$ may contribute to the development of delayed cerebral ischemia after aneurysmal subarachnoid hemorrhage. Transl Stroke Res. 2017;8:165-73.

28. Suzuki H. What is early brain injury? Transl Stroke Res. 2015;6:13.

29. Marbacher S, Fandino J, Kitchen ND. Standard intracranial in vivo animal models of delayed cerebral vasospasm. Br J Neurosurg. 2010;24:415-34.

30. Laban KG, Vergouwen MDI, Dijkhuizen RM, Sena ES, Macleod MR, Rinkel GJE, et al. Effect of endothelin receptor antagonists on clinically relevant outcomes after experimental subarachnoid hemorrhage: a systematic review and meta-analysis. J Cereb Blood Flow Metab. 2015;35:1085-9.

31. Vergouwen MDI, Vermeulen M, van Gijn J, Rinkel GJE, Wijdicks EF, Muizelaar JP, et al. Definition of delayed cerebral ischemia after aneurysmal subarachnoid hemorrhage as an outcome event in clinical trials and observational studies: proposal of a multidisciplinary research group. Stroke. 2010;41:2391-5. 